International Journal of Electrical and Power Engineering 4 (2): 73-77, 2010

ISSN: $1990-7958$

(C) Medwell Journals, 2010

\title{
Movement Consideration from Magnetic Forces Calculation of Rotating Magnetic Bearing
}

\author{
Lotfi Chouikhi, Ammar Tibouche and Antar Bourouina \\ Laboratory of Lamel, Department of Electrical Engineering, University of Jijel, Algeria
}

\begin{abstract}
This study presents the comparative studies between an analytical and numerical calculation of magnetic forces of a radial magnetic bearing. When an unlaminated rotor rotates in a radial magnetic bearing, eddy currents are caused to flow inside the conducting material of the rotor. These eddy currents change the magnetic field of the radial bearing and therefore, the forces on the rotor depend on the eddy currents. Additionally to the levitation force, a tangential force acts on the rotor. When this rotor is excited, the tangential force additionally leads to a cross coupling between the $\mathrm{x}$ and $\mathrm{y}$ axis which may destabilise the system. It will be shown that the forces of a radial magnetic bearing depend on the pole configuration. It can be seen that the calculation of forces depends on the configuration of the system which considered two; NSNS and NNSS.
\end{abstract}

$\underline{\text { Key words: Magnetic force, magnetic bearing, eddy currents, rotational velocity, flux changes, Algeria }}$

\section{INTRODUCTION}

An analytical solution has some advantages compared with numerical results achieved with finite element analysis because it gives a better insight into the problem.

Furthermore, numerical calculations for radial bearings often do not succeed. In order to achieve a correct and stable numerical result a small grid width and a large number of elements is necessary for higher velocity (rotational speed) the number of element increases rapidly and it may reach computational limits (Allaire et al., 1995) however, the analytical methods suffer when the geometry of the design study are more complicated and sometimes the implementation of boundary and flow conditions are practically impossible. In this research, researchers compare the result of magnetic forces, rotational velocity, power losses etc., issued by the two methods and we observe how the flux changes due to eddy currents by FEM.

\section{MATERIALS AND METHODS}

The scheme of the design in reality is shown in Fig. 1 because of its unlaminated rotor it can be transformed on two-dimensional model as shown in Fig. 2.

For the following calculations, all magnetic poles of the radial bearing are assumed to be identical. In order to solve Maxwell's equations it is necessary to define appropriate boundary conditions. The magnetic flux density at the boundary between the stator and the

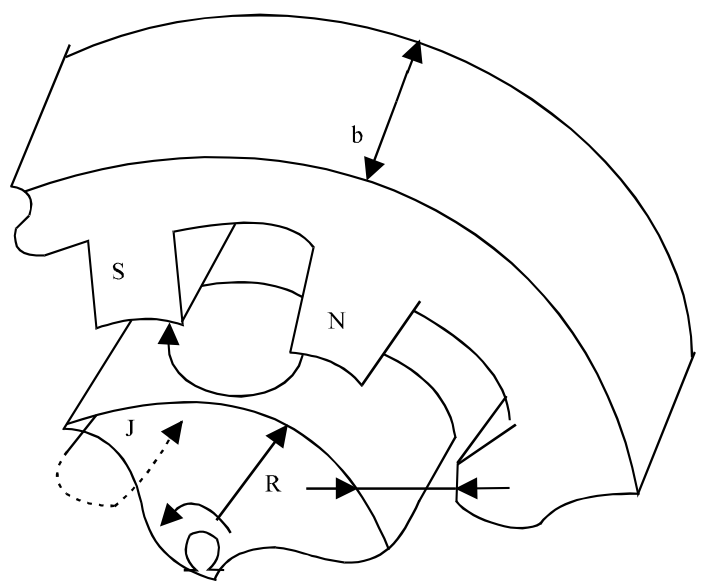

Fig. 1: Cross-section of a radial magnetic bearing

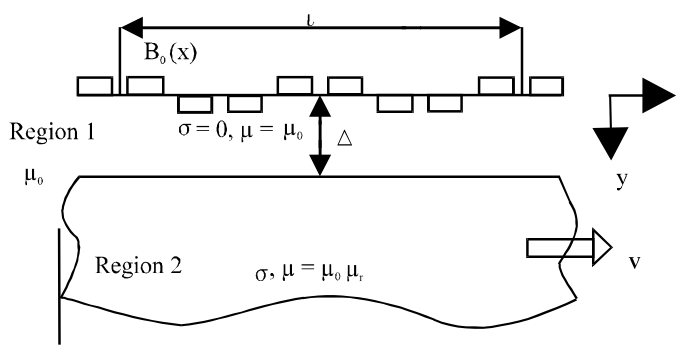

Fig. 2: Moving conducting medium under the applied flux density Bo

Corresponding Author: Lotfi Chouikhi, Laboratory of Lamel, Department of Electrical Engineering, University of Jijel, Algeria 
air gap, conductivity and permeability are assumed to be constant. The applied flux density is periodic and hysteresis effect are neglected. Another assumption is that the magnetic field and induced currents tend to concentrate at the surface of the rotor. When the penetration depth $\delta=(2 /(\sigma \mu \Omega))^{0.5}$ of the magnetic field is small compared with the diameter of the rotor the error using cartesian coordinates $(x, y)$ instead of cylindrical coordinate $(r, \varphi)$ is small.

The rotational speed $\Omega$ can then be replaced by the velocity $V x=\Omega / R$, hence the problem of a rotor rotating in radial bearing can be approximated by a semi-infinite conducting plate moving under magnetic poles.

\section{RESULTS AND DISCUSSION}

Fourier approximation: In the first step, the flux density will be approximated by a Fourier series (Marko, 1986).

$$
\begin{aligned}
& \mathrm{B}_{0}=\sum_{\mathrm{n}=1}^{\infty} \mathrm{A}_{\mathrm{n}} \cos \left(\mathrm{k}_{\mathrm{n}} \mathrm{x}\right)+\mathrm{B}_{\mathrm{n}} \sin \left(\mathrm{k}_{\mathrm{n}} \mathrm{x}\right) \\
& \mathrm{B}_{0}=\sum_{\mathrm{n}=1}^{\infty} \frac{\mathrm{A}_{\mathrm{n}}-\mathrm{j} \mathrm{B}_{\mathrm{n}}}{2} \mathrm{e}^{\mathrm{j} \mathrm{k}_{\mathrm{n}} \mathrm{x}}+\frac{\mathrm{A}_{\mathrm{n}}+j \mathrm{~B}_{\mathrm{n}}}{2} \mathrm{e}^{-\mathrm{j} \mathrm{k}_{\mathrm{n}} \mathrm{x}} \\
& \mathrm{B}_{0}=\sum \mathrm{C}_{\mathrm{n}} \mathrm{e}^{\mathrm{j} \mathrm{k}_{\mathrm{n}} \mathrm{x}}+\bar{C}_{\mathrm{n}} \mathrm{e}^{-j \mathrm{k}_{\mathrm{n}} \mathrm{x}}
\end{aligned}
$$

The representation of flux density and its development in Fourier's series transform for the two configurations (NNSS and NSNS) are shown in Fig. 3 and 4. Comparing the two Fourier series are shown in Fig. 3 and 4, it can seen that the configurations NNSS have more harmonic components than NSNS one.

Magnetic fields: In this study, all calculations will be shown for one term $\mathrm{Bo}=\mathrm{Cx} \exp (\mathrm{jKx})$ of the harmonic components but the final result is the sum of each components (superposition theorem).

Maxwell's equations without time variant fields can be combined to have second order partial differential equations as:

$$
\begin{aligned}
& \nabla \mathrm{xE}=0 \\
& \nabla \mathrm{xB}=\mathrm{J}
\end{aligned}
$$

The material equation is:

$$
\mathrm{B}=\mu \mathrm{H}=\mu_{0} \mu_{\mathrm{r}} \mathrm{H}
$$

Ohm's law is given by:
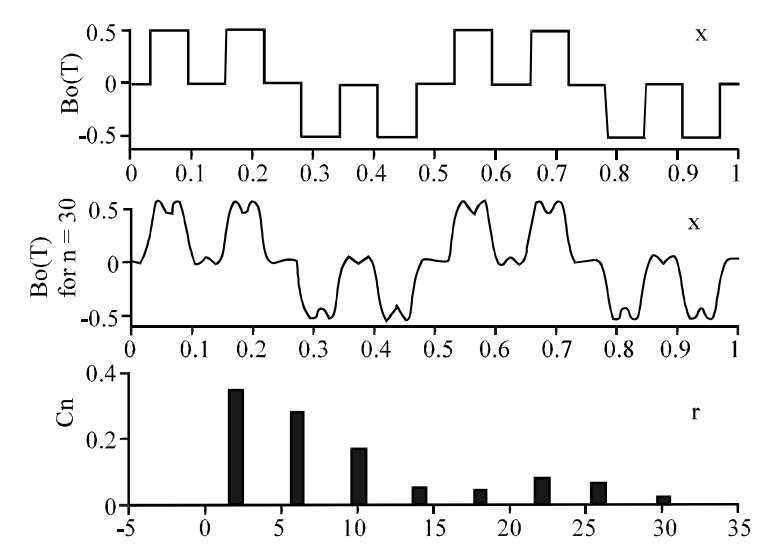

Fig. 3: Approximation and development in Fourier's series of flux density $\mathrm{Bo}(\mathrm{x})$ of NNSS configuration
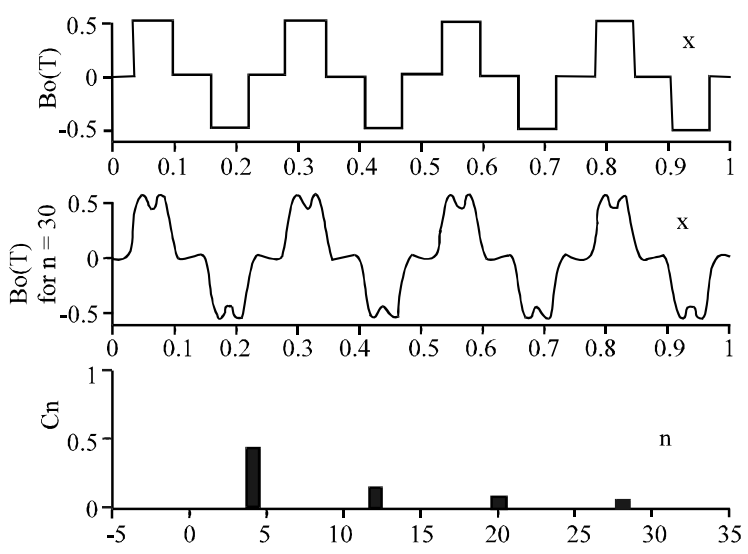

Fig. 4: Approximation and development in Fourier's series of flux density $\mathrm{Bo}(\mathrm{x})$ of NSNS configuration

$$
J=\sigma(E+v x B)
$$

Equation 2-4 can be combined to second order partial differential equations:

$$
\frac{1}{\mu \sigma} \nabla^{2} \mathrm{~B}+\nabla \times(\mathrm{vxB})=0
$$

The flux density has components in $\mathrm{x}$ and $\mathrm{y}$ directions. Conductive part is moving in $\mathrm{x}$ direction:

$$
\mathrm{B}=\left(\begin{array}{l}
\mathrm{B}_{\mathrm{z}} \\
\mathrm{B}_{\mathrm{y}} \\
0
\end{array}\right), \mathrm{J}=\left(\begin{array}{l}
0 \\
0 \\
\mathrm{~J}_{\mathrm{z}}
\end{array}\right), \mathrm{v}=\left(\begin{array}{l}
\mathrm{v}_{\mathrm{z}} \\
0 \\
0
\end{array}\right)
$$

$\mathrm{B}_{\mathrm{z}}, \mathrm{B}_{\mathrm{y}}$ and $\mathrm{J}_{z}$ are function of $\mathrm{x}$ and $\mathrm{y}$. Conbining Eq. 5 and 6 leads to: 


$$
\begin{aligned}
& \frac{\partial^{2} \mathrm{~B}_{\mathrm{x}}}{\partial \mathrm{x}^{2}}+\frac{\partial^{2} \mathrm{~B}_{\mathrm{x}}}{\partial \mathrm{y}}-\mu \delta \mathrm{v}_{\mathrm{x}} \frac{\partial \mathrm{B}_{\mathrm{x}}}{\partial \mathrm{x}}=0 \\
& \frac{\partial^{2} \mathrm{~B}_{\mathrm{y}}}{\partial \mathrm{x}^{2}}+\frac{\partial \mathrm{B}_{\mathrm{y}}}{\partial \mathrm{y}^{2}}-\mu \delta \mathrm{v}_{\mathrm{x}} \frac{\partial \mathrm{B}_{\mathrm{y}}}{\partial \mathrm{x}}=0
\end{aligned}
$$

The magnetic flux density is driven by the applied magnetic field density Bo. Hence, the solutions with the same travelling ware dependence on $\mathrm{x}$ are assumed and the flux density takes the form:

$$
\mathrm{B}(\mathrm{x}, \mathrm{y})=\overline{\mathrm{B}}(\mathrm{y}) \mathrm{e}^{\mathrm{jkx}}
$$

The Eq. 7 can be transformed to:

$$
\begin{aligned}
& \frac{\partial^{2} \bar{B}_{x}}{\partial y_{2}}-q^{2} \bar{B}_{z}=0 \\
& \frac{\partial^{2} \bar{B}_{y}}{\partial y^{2}}-q^{2} \bar{B}_{y}=0
\end{aligned}
$$

With:

$$
\mathrm{q}=\sqrt{\mathrm{k}^{2}+\mathrm{k} \sigma \mu \mathrm{v}_{\mathrm{x}}}
$$

The solution domain is divided into two regions:

Region 1: Corresponding to the air gap $(\sigma=0)$

Region 2: Corresponding to the moving conducting medium.

Forces calculation: To calculate the forces acting on the conducting medium we use Maxwell's stress tensor (Jufer, 1979), the tangential force; corresponding to $x$ and the normal force; corresponding to $y$ are expressed as:

$$
\begin{aligned}
& \mathrm{F}_{\mathrm{x}}=-\frac{1}{\mu_{0}} \oint_{\mathrm{s}} \mathrm{B}_{\mathrm{x}} \mathrm{B}_{\mathrm{y}} \mathrm{da} \\
& \mathrm{F}_{\mathrm{y}}=-\frac{1}{2 \mu_{0}} \oint\left(\mathrm{B}_{\mathrm{y}}^{2}-\mathrm{B}_{\mathrm{x}}^{2}\right) \mathrm{da}
\end{aligned}
$$

where $\mathrm{a}$ is any closed surface surrounding the conducting body. It's useful to choose the surface at $y=\Delta$ and integrate $\mathrm{x}$ from 0 to $2 \pi$ and $\mathrm{y}$ from 0 to $\mathrm{b}$, where $\mathrm{b}$ is the longitudinal length Fig. 1. After several stage of calculation the forces will have the following shape:

$$
\begin{aligned}
& \mathrm{F}_{\mathrm{x}}=\frac{\mathrm{jbu}}{\mu_{0}} \sum_{\mathrm{n}=1}^{\infty} \frac{\mathrm{C}_{\mathrm{n}} \overline{\mathrm{C}}_{\mathrm{n}}\left(\mathrm{q}_{\mathrm{n}}-\overline{\mathrm{q}}_{\mathrm{n}}\right)}{\gamma_{\mathrm{n}} \bar{\gamma}_{\mathrm{n}} \mu_{\mathrm{r}} \mathrm{k}_{\mathrm{n}}} \\
& \mathrm{F}_{\mathrm{y}}=\frac{\mathrm{bu}}{\mu_{0}} \sum_{\mathrm{n}=1}^{\infty} \frac{\mathrm{C}_{\mathrm{n}} \overline{\mathrm{C}}_{\mathrm{n}}}{\gamma_{\mathrm{n}} \bar{\gamma}_{\mathrm{n}}} \frac{\mathrm{q}_{\mathrm{n}} \overline{\mathrm{q}}_{\mathrm{n}}-\mu_{\mathrm{r}}{ }^{2} \mathrm{k}_{\mathrm{x}}^{2}}{\mu_{\mathrm{r}} \mathrm{k}_{\mathrm{n}}^{2}}
\end{aligned}
$$

$\overline{\mathrm{C}}_{\mathrm{n}}, \overline{\mathrm{q}}_{\mathrm{n}}$ and $\bar{\gamma}_{\mathrm{n}}$ are the conjugate complex values of $\mathrm{C}_{\mathrm{n}}, \mathrm{q}_{\mathrm{n}}$ and $\gamma_{n}$. The subscript $n$ refers to the $n$-th harmonic of the applied flux density series. It is interest to see the variation of force as function of velocity Fig. 5 . We note that the levitation force Fy contrary to drag force Fx decrease when velocity increase and this is a major inconvenient because we search always to save the force of levitation as constant as possible. If we compare the two configurations we can classify the NSNS as the best on account of low variation of forces caused by velocity increase.

Moreover to the first advantage, we can see also that the NSNS configuration have the least power losses quantities ( $\mathrm{P}=\mathrm{v} . \mathrm{Fx})$ compared to NNSS configuration (Ahrens and Kucera, 1996) (Fig. 6). For axisymetrical calculation we consider a real model as shown in Fig. 1 in this case, the reaction of excited

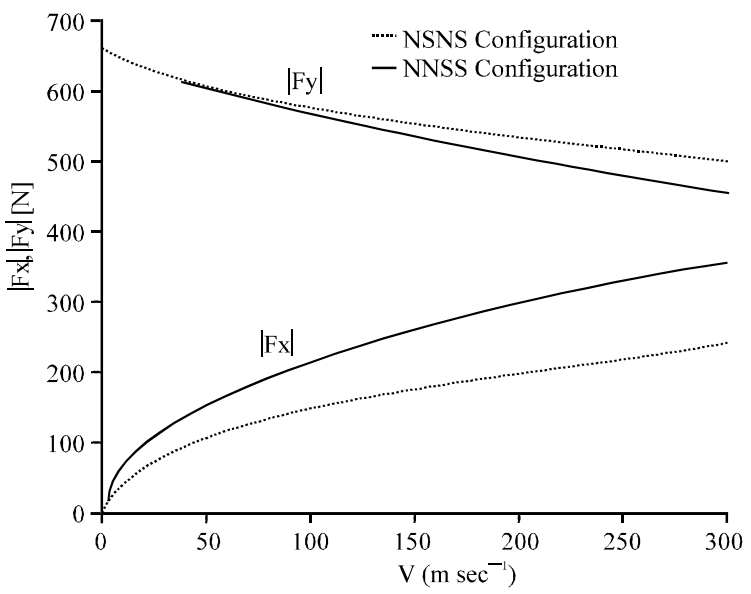

Fig. 5: Forces as function of linear velocity, upper curves: levitation force Fy, lower curves; drag force $\mathrm{Fx}$

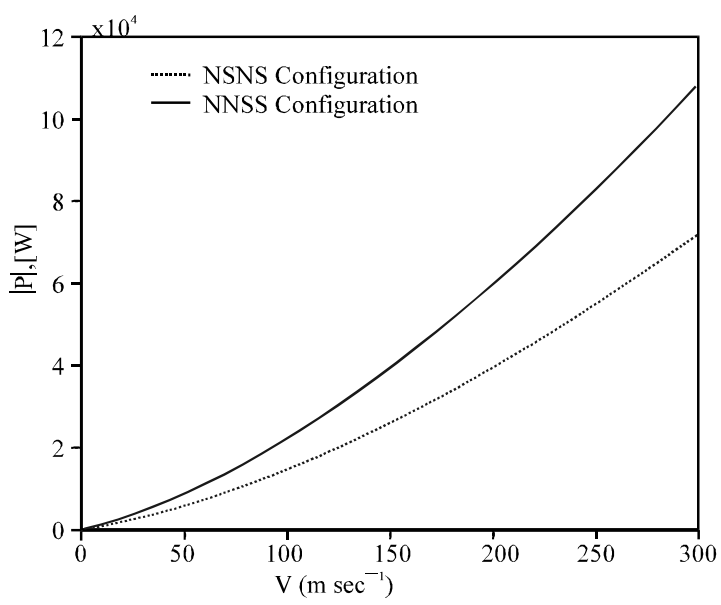

Fig. 6: Power losses as function of linear velocity 

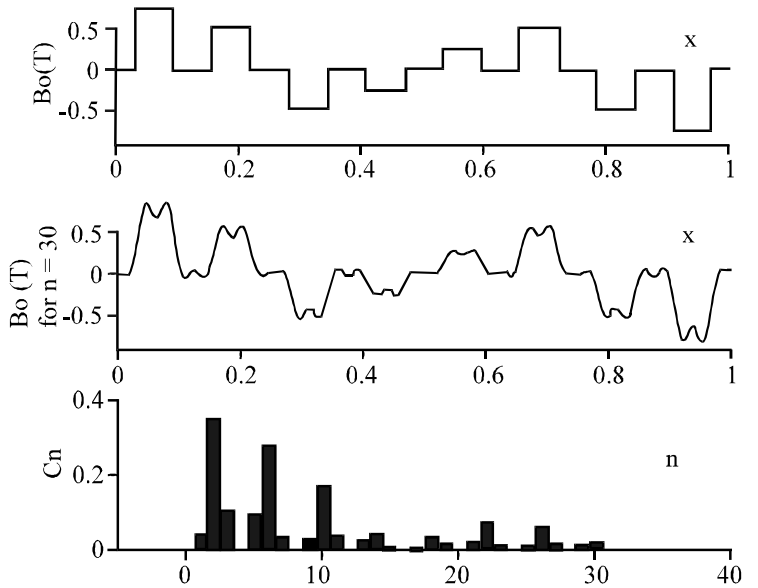

Fig. 7: Approximation and development in Fourier's series of flux density $\mathrm{Bo}(\mathrm{x})$ of NNSS configuration in axisymetrical case

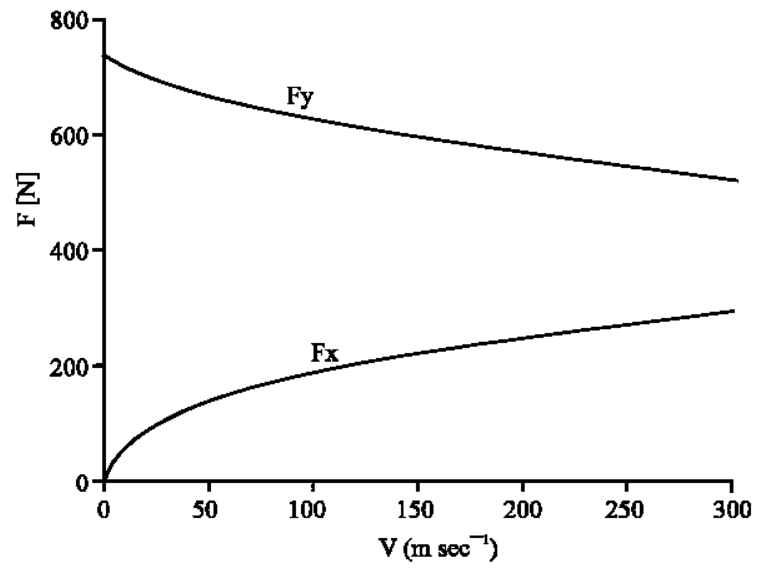

Fig. 8: Forces as function of linear velocity

rotor cause a change in flux density, therefore the new flux density source is shown in Fig. 7. Respecting the conservation low of the total energy of a system (Ahrens and Kucera, 1996). The result of forces is shown in Fig. 8, in which we see the same phenomenon's when we have taken the cartésian model Fig. 5.

Numerical application: Numerical application is realized for confirming the result obtained by analytical one. In the calculation, we use the same formulation as Eq. 5 in term of magnetic vector potential $A$. The source variation is considered for respecting the variation of flux density Fig. 7. The solution expressed by vector potential $\mathrm{A}$ is shown in Fig. 9 and 10. For the two values of velocity (10 and $300 \mathrm{~m} \mathrm{sec}^{-1}$ ). From Fig. 11 and 12, we can say that the results concerning the values of forces and power losses are suitable and confirm enormously with those obtained by analytical approach.

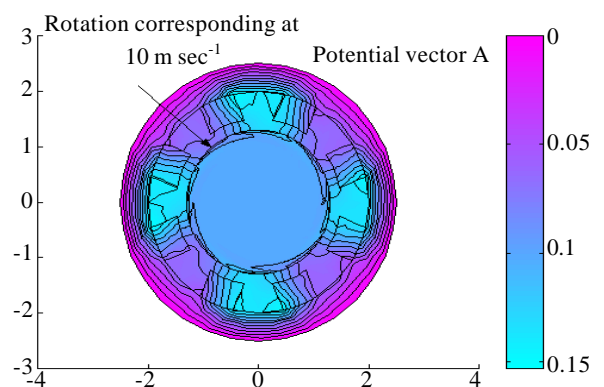

Fig. 9: Potential vector $A$ at $\mathrm{v}=10 \mathrm{~m} \mathrm{sec}^{-1}$

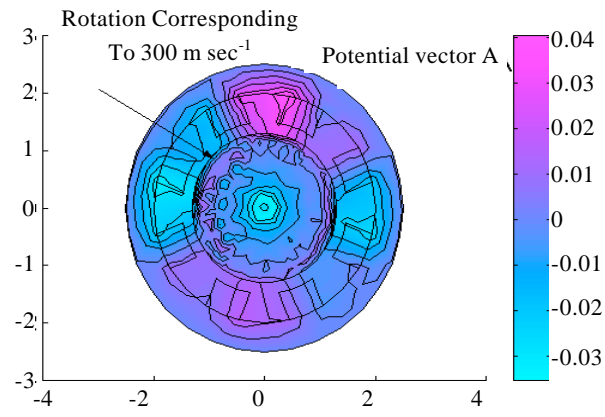

Fig. 10: Potential vector $\mathrm{A}$ at $\mathrm{v}=300 \mathrm{~m} \mathrm{sec}^{-1}$

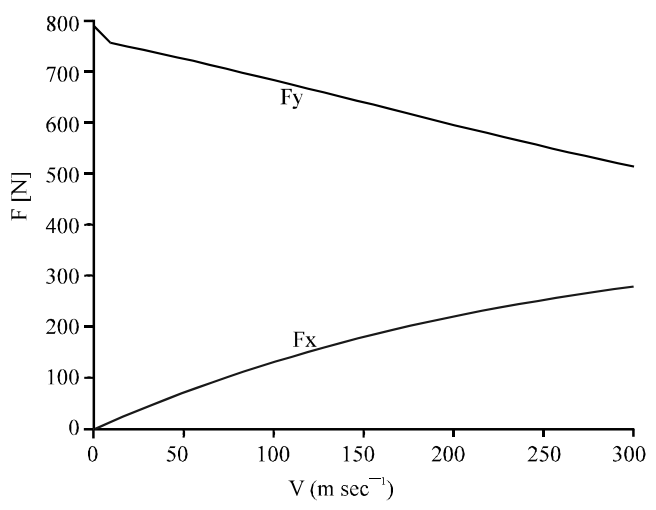

Fig. 11: Forces as function of linear velocity (Numerical results)

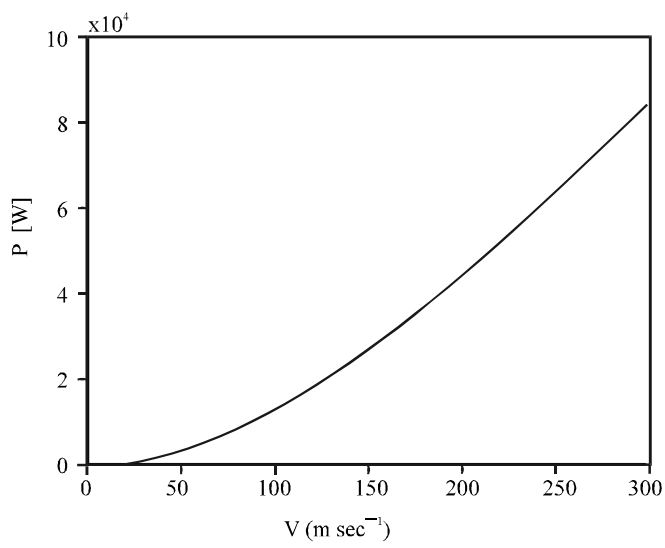

Fig. 12: Power losses as function of linear velocity 


\section{CONCLUSION}

This study shows a model for eddy currents in an unlaminated rotor, rotating inside a radial magnetic bearing. The levitational force and the drag force acting on the rotor is calculated using analytical and numerical methods in which we have seen:

- The advantage of NSNS configuration compared with NNSS one

- How to introduce the change of flux density caused by excited rotor in axisymetrical approach

- The confirmation of analytical results by obtaining a comparable and significant numerical one

\section{REFERENCES}

Ahrens, M. and L. Kucera, 1996. Analytical calculation of fields, forces and losses of a radial magnetic bearing with rotating rotor considering eddy currents. Proceedings of the 5th International Symposium on Magnetic Bearings, Aug. 28-30, Kanazawa, pp: 253-258.

Allaire, P., R. Rockwell and M. Kasarda, 1995. Magnetic and electric fields equations for magnetic bearing applications. MAG'95 Magnetic Bearings, Magnetic Drives and Dry Gas Seals Conference and Ex-hibition, Alexandria.

Jufer, M., 1979. Electromecanique, Traite d'electricite, d'electronique et d'electrotechnique. Editions Georgi.

Marko, H., 1986. Methoden der Systemtheorie. SpringerVerlag, Berlin. 